\title{
Capsule Commentary on Leykum et al., the Association Between Sensemaking During Physician Team Rounds and Hospitalized Patients' Outcomes
}

\author{
Cordelia R. Stearns, MD \\ Department of Medicine, Highland Hospital, Alameda Health System, Oakland, CA, USA.
}

J Gen Intern Med 30(12): 1853

DOI: $10.1007 / \mathrm{s} 11606-015-3398-Z$

(c) Society of General Internal Medicine 2015

$\mathrm{T}$ oday's inpatient providers are caring for ever-sicker patients during shorter hospitalizations, with reduced resident duty hours, while leading the charge for quality improvement and value-based care. ${ }^{1}$ In this changing clinical landscape, the daily routines of inpatient teams must be fine-tuned to optimize efficiency, reliability, and safety.

Clinical reasoning is often used as the framework for discussions of how providers conceptualize and treat individual patients, but it can oversimplify or neglect the reality of the many competing tasks carried out by providers on busy services. ${ }^{2}$ This study by Leykum et al. ${ }^{3}$ introduces the concept of "sensemaking" as a tool for inpatient teams to use in organizing and carrying out their daily tasks. The authors used measures of sensemaking to assess how teams prioritized, processed, and cared for multiple sick patients simultaneously, and found that higher markers of sensemaking were associated with improved patient outcomes, including fewer complications and shorter lengths of stay.

In many ways, the most surprising finding of the study was just how infrequently the low bar for sensemaking was met. The majority of observed teams never conducted rounds "purposefully," as defined by the authors, and most teams had low sensemaking scores when rated on discussions of complex patients. It is not hard to imagine the many possible differences between teams with high and low sensemaking scores; teams that are explicit, flexible, and purposeful in daily work might also communicate more effectively both with other providers and with their patients, be more responsive to feedback, and be open to outside ideas. The question that remains is whether interventions to encourage sensemaking would make a difference in patient outcomes for teams that did not naturally function this way.

There is a growing body of literature on how rounding structure can affect resident education in the new duty-hours climate. ${ }^{4,5}$ As we consider how this core of our daily work affects trainees and patients alike, sensemaking may be a foundation around which we can evaluate and refine our practice. The authors can now investigate whether teams with low sensemaking scores are able to improve patient outcomes by improving these measures of sensemaking.

Conflicts of Interest: The author has no conflict with any of the material in this manuscript.

Corresponding Author: Cordelia R. Stearns, MD; Department of Medicine Highland Hospital, Alameda Health System, Oakland, CA, USA (e-mail: cstearns@alamedahealthsystem.org).

\section{REFERENCES}

1. Wachter RM. The hospitalist field turns 15: new opportunities and challenges. J Hosp Med. 2011;6(4):E1-4.

2. Carrier A, Levasseur M, Freeman A, Mullins G, Quenec'hdu S, Lalonde $\mathbf{L}$, Gagnon M, Lacasse F. Influence of societal and practice contexts on professionals' clinical reasoning: a scoping study protocol. Br Med J Open. 2013;3(4), e002887.

3. Leykum L, Chesser H, Lanham HJ, Pezzia C, Palmer R, Ratcliffe T, Reisinger H, Agar M, and Pugh J. The association between sensemaking during physician team rounds and hospitalized patients' outcomes. J Gen Intern Med. 2015. (Article SPI \# 3377).

4. Reilly J, Bennett N, Fosnocht K, Williams K, Kangovi S, Jackson R, Ende J. Redesigning rounds: towards a more purposeful approach to inpatient teaching and learning. Acad Med. 2015;90(4):450-453.

5. Shoeb M, Khanna R, Fang $\mathbf{M}$, Sharpe B, Finn K, Ranji S, Monsah B. Internal medicine rounding practices and the accreditation council for graduate medical education core competencies. J Hosp Med. 2014;9(4):239-243

This comment refers to the article available at: http://dx.doi.org/10.1007/ s11606-015-3196-7.

Published online July 10, 2015 